for initiation of conduction block developed using the simpler models need only be adjusted, not discarded, when considering a more anatomically complex model. This result builds on previous studies by this group and by others in which simple onedimensional models of cardiac fibers have been extended first to two-dimensional sheets of cardiac tissue and then to threedimensional slabs (Figure 1). The present study extends these models further by incorporating important aspects of gross and microscopic cardiac anatomy, including regional differences in fiber orientation. Despite the increased complexity of the anatomical substrate, the role of APD restitution in the development of VF remains intact, in that steep restitution is necessary for the initiation of wavebreak and for the disintegration of a single spiral wave into multiple wavelets (see Figures 4 and 6 from Xie et al. [ref. 14]).

Although the results of Xie et al. underscore the importance of electrical restitution for the development of VF in a more anatomically correct heart, the next set of questions inevitably arises regarding the potential impact of anatomically related electrophysiological features of the heart that were not included in their model, such as transmural and base-to-apex gradients of electrical properties, the presence of a His-Purkinje system, microscopic muscle bundle architecture, etc. The relative impact of cardiac contraction, the auto- nomic nervous system, and the anatomical, electrical, and mechanical remodeling that occur in response to the demands created by various forms of heart disease also remain to be addressed (Figure 1).

Although the prospect of accommodating all of the complexities cited above into a single model of the heart might seem daunting, such models are under development by several groups (e.g., see refs. 15, 16), and with the exponential improvement in computing power over time and the healthy interest of biologists and computer scientists, such problems promise to become tractable. It will be interesting, however, to discover how much better we understand the development of ventricular arrhythmias as layers of complexity are added. Perhaps we will be able to look back on the present state of the art and find that all of the important pieces already were in place - or perhaps not.

Address correspondence to: Robert F. Gilmour, Jr., Department of Biomedical Sciences, Cornell University, Ithaca, New York 14853-6401, USA. E-mail: rfg2@cornell.edu.

1. Zipes, D.P., and Wellens, H.J. 1998. Sudden cardiac death. Circulation. 98:2334-2351.

2. Janse, M.J. 1986. Reentry rhythms. In The heart and cardiovascular system. H.A. Fozzard, et al., editors. Raven Press. New York, New York, USA. 1203-1238.

3. Winfree, A.T. 1998. Evolving perspectives during 12 years of electrical turbulence. Chaos. 8:1-20.

4. Antzelevitch, C. 2000. Electrical heterogeneity, cardiac arrhythmias, and the sodium channel. Circ. Res. 87:964-965.
5. Janse, J., and Wit, A.L. 1989. Electrophysiological mechanisms of ventricular arrhythmias resulting from myocardial ischemia and infarction. Physiol. Rev. 69:1049-1169.

6. Watanabe, M.A., Fenton, F.H., Evans, S.J., Hastings, H.M., and Karma, A. 2001. Mechanisms for discordant alternans. J. Cardiovasc. Electrophysiol. 12:196-206.

7. Qu, Z., Garfinkel, A., Chen, P-S., and Weiss, J.N. 2000. Mechanisms of discordant alternans and induction of reentry in simulated cardiac tissue. Circulation. 102:1664-1670

8. Fox, J.J., Riccio, M.L., Hua, F., Bodenschatz, E., and Gilmour, R.F., Jr. 2002. Spatiotemporal transition to conduction block in canine ventricle. Circ. Res. 90:289-296.

9. Fox, J.J., Riccio, D.L., Drury, P., Werthman, A., and Gilmour, R.F., Jr. 2003. Dynamic mechanism for conduction block in heart tissue. New J. Phys. 5:101.1-101.14.

10. Nolasco, J.B., and Dahlen, R.W. 1968. A graphic method for the study of alternation in cardiac action potentials. J. Appl. Physiol. 25:191-196.

11. Karma, A. 1993. Spiral breakup in model equations of action potential propagation in cardiac tissue. Phys. Rev. Lett. 71:1103-1106.

12. Rosenbaum, D.S., et al. 1994. Electrical alternans and vulnerability to ventricular arrhythmias. $N$. Engl. J. Med. 330:235-241.

13. Gilmour, R.F., Jr., and Chialvo, D.R. 1999. Electrical restitution, critical mass, and the riddle of fibrillation. J. Cardiovasc. Electrophysiol. 10:1087-1089.

14. Xie, F., et al. 2004. A simulation study of the effects of cardiac anatomy in ventricular fibrillation. J. Clin. Invest. 113:686-693. doi:10.1172/JCI200417341.

15. Hunter, P.J., Pullan, A.J., and Smaill, B.H. 2003. Modeling total heart function. Annu. Rev. Biomed. Eng. 5:147-77.

16. Noble, D. 2002. Modeling the heart-from genes to cells to the whole organ. Science. 295:1678-1682.

17. Garfinkel, A., et al. 2000. Preventing ventricular fibrillation by flattening cardiac restitution. Proc. Natl. Acad. Sci. U. S. A. 97:6061-6066.

18. Mareib, E.N., and Branstrom, M.J. 1996. InterActive physiology: cardiovascular system [CD-ROM]. Benjamin/Cummings. San Francisco, California, USA.

\title{
Immune system versus tumor: shifting the balance in favor of DCs and effective immunity
}

\author{
Howard L. Kaufman'1 and Mary L. Disis ${ }^{2}$ \\ 1Department of Surgery, Columbia University, New York, New York, USA. ${ }^{2}$ Tumor Vaccine Group, University of Washington, Seattle, Washington, USA.
}

\begin{abstract}
Despite the initial excitement over cancer vaccines, the clinical effectiveness of immunotherapy has been disappointing. The suppressive milieu present within established tumors inhibits effective immune responses, although new strategies are emerging to manipulate the local tumor microenvironment and shift the balance back to a proinflammatory environment, promote DC activation, and enhance tumor immunity (see the related article beginning on page 774).
\end{abstract}

Nonstandard abbreviations used: CC chemokine receptor 7 (CCR7); human papillomavirus (HPV); Toll-like receptor (TLR).

Conflict of interest: The authors have declared that no conflict of interest exists.

Citation for this article:

J. Clin. Invest. 113:664-667 (2004).

doi:10.1172/JCI200421148.
The balance between host antitumor immunity and tumor escape

Therapeutic vaccines that have targeted established disease in cancer patients have not been successful in eliciting significant, long-lasting tumor regression. Over the last several decades most attempts to vaccinate against cancer and generate an antitumor response have been in patients with measurable tumors, and the clinical endpoint of such trials has been to evaluate a reduction in tumor burden. The inability to effectively decrease tumor growth with active immunization is most likely due, in large part, to an unfavorable tumor microenvironment incapable of propagating a robust immune response (1).

The defects in the tumor environment are many (Figure 1). The tumor itself can secrete a variety of substances that will depress or inhibit local immunity, such as IL- 6 or TGF- $\beta$, 


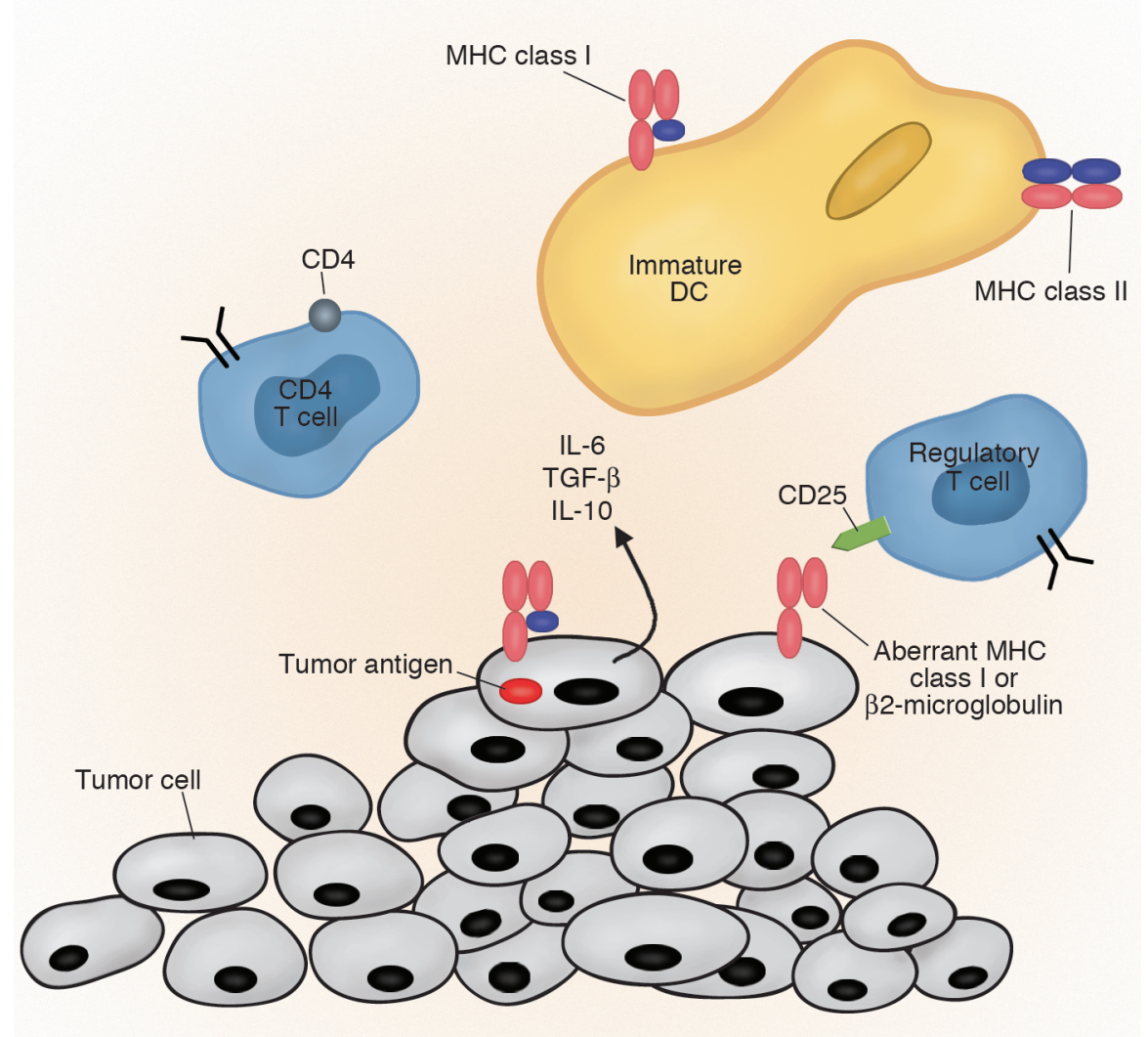

which can stop the proliferation of antigenspecific $T$ cells. Tumors can lose the expression of both MHC and costimulatory molecules, making them invincible to $\mathrm{T}$ cell attack (2). The tumor microenvironment can also be adversely affected by the phenotype of local infiltrating $T$ cells. T cell subsets that limit the generation of tumor-specific immunity are abundant in cancer patients. Investigations of preexistent tumorspecific $\mathrm{T}$ cell immunity in patients with renal cell carcinoma have demonstrated that those patients living with disease were more likely to have tumor-specific Th2 responses, whereas those patients with no evidence of disease, i.e., successfully treated, were more likely to have Th1-specific immunity directed against renal cell cancer antigens (3). Furthermore, $\mathrm{CD}_{25} 5^{+} \mathrm{T}$ regulatory cells are abundant in both the peripheral blood and tumors of patients with cancer (4). These regulatory $T$ cells have been shown to dampen the proliferation of cancer-specific cytotoxic $T$ cells by direct contact with antigenprimed $\mathrm{T}$ cells and through elaboration of immunosuppressive cytokines such as TGF- $\beta$ (5). The active inhibition of a local inflammatory response by $\mathrm{T}$ cell subsets adds to the immune inhibitory cytokine milieu that characterizes most human cancers.

\section{The local tumor environment may suppress DC function and promote tolerance}

The definition over the last decade of a multitude of tumor antigens has underscored the importance of creating a proinflammatory immune response to eradicate cancer. The majority of tumor antigens identified have been self-proteins, and tolerance has emerged as an important mechanism of tumor immune escape. Thus, methods of generating anticancer immunity must focus on the initiation and maintenance of an inflammatory environment complete with APCs potent enough to stimulate naive $T$ cells, i.e., DCs $(6,7)$. The potential of DCs in eliciting antitumor immunity has been an area of intense investigation. DCs are unique in their expression of important costimulatory molecules, the elaboration of proinflammatory cytokines, and the ability to process and present self-antigens (Figure 2). The clinical application of DCs in the form of cancer vaccines, however, has been only moderately successful (8). The majority of clinical approaches have used ex vivo-derived DCs to elicit tumor-specific immunity. However, cultured DCs have demonstrated poor homing to lymph nodes once injected in vivo, inconsistent processing and presentation of anti-

\section{Figure 1}

Tumors may escape immune detection through a variety of mechanisms that create a local microenvironment unfavorable for effective tumor immunity. Local DCs may be immature and unable to take up, process, or present antigens. These DCs may also be inhibited from migrating to regional lymph nodes or may actually induce tolerance, especially when presenting self-antigens. The tumor site may also be infiltrated with regulatory $T$ cells that are able to mediate suppression of antigen-primed T cells. The helper CD4 T cell response may also be skewed toward a Th2 phenotype, which inhibits the initiation of Th1 T cells and effective cellular immunity. The tumor cells may express aberrant $\mathrm{MHC}$ class I molecules or $\beta 2$-microglobulin, resulting in inadequate antigen presentation and, thus, inefficient recognition of tumors by effector T cells. Finally, tumor cells and the surrounding stroma may release a number of suppressive cytokines, such as IL-6, IL-10, and TGF- $\beta$. This creates an environment that is not conducive to local immunity, which allows tumor cells to escape. gen, and, depending on their maturation status, the ability to either enhance or inhibit the generation of tumor antigen-specific immunity (9). In this issue of the JCI, Furumoto and colleagues present a novel approach to overcoming the limitations of ex vivo-derived DCs and in doing so outline a strategy potent enough to influence the local immune microenvironment in an established tumor, which supports the initiation and propagation of an effective tumor-specific immune response (10). They demonstrated that the local presence of CC chemokine ligand 20 within immunogenic CT26 tumors attracted a large number of DCs to the tumor site and could mediate tumor regression. However, poorly immunogenic B16 tumors were not rejected unless local CpG was also injected. This provides a method for manipulating both the number of local DCs and the local environment, resulting in effective tumor immunity.

\section{Manipulating the local tumor environment may promote DC activation and enhance host immune responses}

Stimulating DCs in vivo may allow natural maturation and retention of their most potent functions and represents a more 

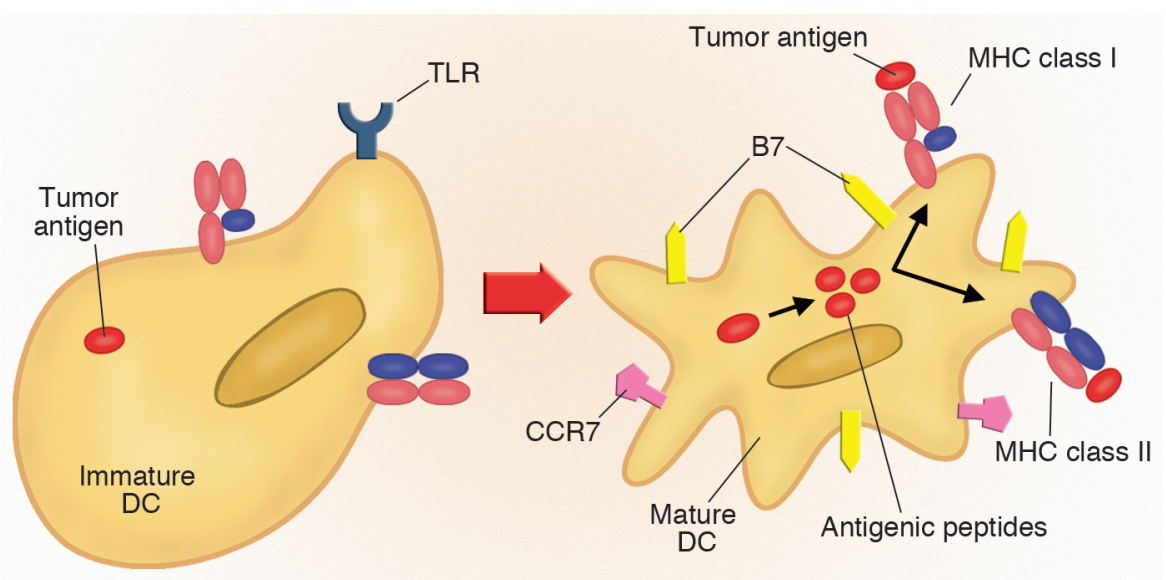

Figure 2

DCs exist primarily in two distinct phenotypic forms. Immature DCs are able to circulate through peripheral tissues and sample soluble antigen and possibly necrotic or apoptotic cells. These DCs express few costimulatory molecules and are unable to prime T cell responses. Once DCs begin to process and present antigen they "mature" into a more stellate shape capable of presenting antigen and initiating an adaptive immune response. The presence of TLRs allows the DC to recognize common molecular patterns found on a variety of pathogenic organisms, so-called pathogenassociated molecular patterns. TLR signaling is thought to represent a major mechanism of innate immunity and is another process that results in APC conditioning and release of proinflammatory cytokines. Maturation of DCs also results in increased expression of MHC, costimulatory molecules, and chemokine receptors that direct DCs to regional lymph nodes where they are more likely to encounter their cognate $\mathrm{T}$ cell and are able to prime $\mathrm{T}$ cell responses.

rational approach to immunotherapy. The migration of DCs, as well as other immune cells, is mediated by chemokines through a concentration-dependent process attracting cells to sites of chemokine secretion (11). Recent evidence suggests that chemokines induce motility, and possibly other proinflammatory and activating processes, through the selected expression of specific membrane receptors (12). These receptors may be differentially regulated during different stages of cellular differentiation. For example, immature DCs circulate through peripheral tissues where they can acquire antigen at sites of inflammation. As DCs mature, they increase their expression of the CC chemokine receptor 7 (CCR7), which directs their migration to regional lymph

\section{Figure 3}

Following successful immunization, the tumor microenvironment is dramatically altered. DC maturation occurs, and the DCs are induced to express strong costimulatory molecules able to effectively prime CD4 and CD8 T cell responses. When CD4 T cells are skewed to a Th1 phenotype, they further promote $T$ cell priming and alter the local cytokine environment by releasing proinflammatory cytokines, such as IFN- $\gamma$ and IL-12. This environment is favorable for tumor cell recognition and results in tumor rejection. nodes where constitutively secreted chemokines attract mature DCs via the CCR7 receptor. Since naive $T$ cells also express CCR7, the nodal chemokines orchestrate the co-localization of antigen- presenting DCs with $\mathrm{T}$ cells, thus increasing the likelihood of DC-T cell interaction and $\mathrm{T}$ cell priming.

Several animal models have already demonstrated that chemokine expression within tumors can induce the migration of $\mathrm{T}$ cells and APCs to those sites and can mediate modest antitumor responses $(13,14)$. Thus, local expression of chemokines can alter the types of cell present within tumors. Once appropriate cells are mobilized, they can be further activated or expanded by the use of other potent immune stimulatory molecules, such as cytokines and costimulatory molecules. In the case of DCs this allows for uptake and presentation of tumor antigens directly from established tumors, avoiding the need for defined antigens. Furthermore, the DC can be activated at the site of maturation through the co-expression of local costimulatory molecules, proinflammatory cytokines, and Tolllike receptor (TLR) agonists (Figure 3 ). The ability to manipulate the type of cells, their maturation status, and functional activity at specific sites may introduce a new therapeutic strategy for manipulation of host immune responses against tumors.

Immunization in the setting of cancer prevention or even in minimal residual disease is now beginning to demonstrate that if the immune-dampening effects of the tumor microenvironment are not operative,

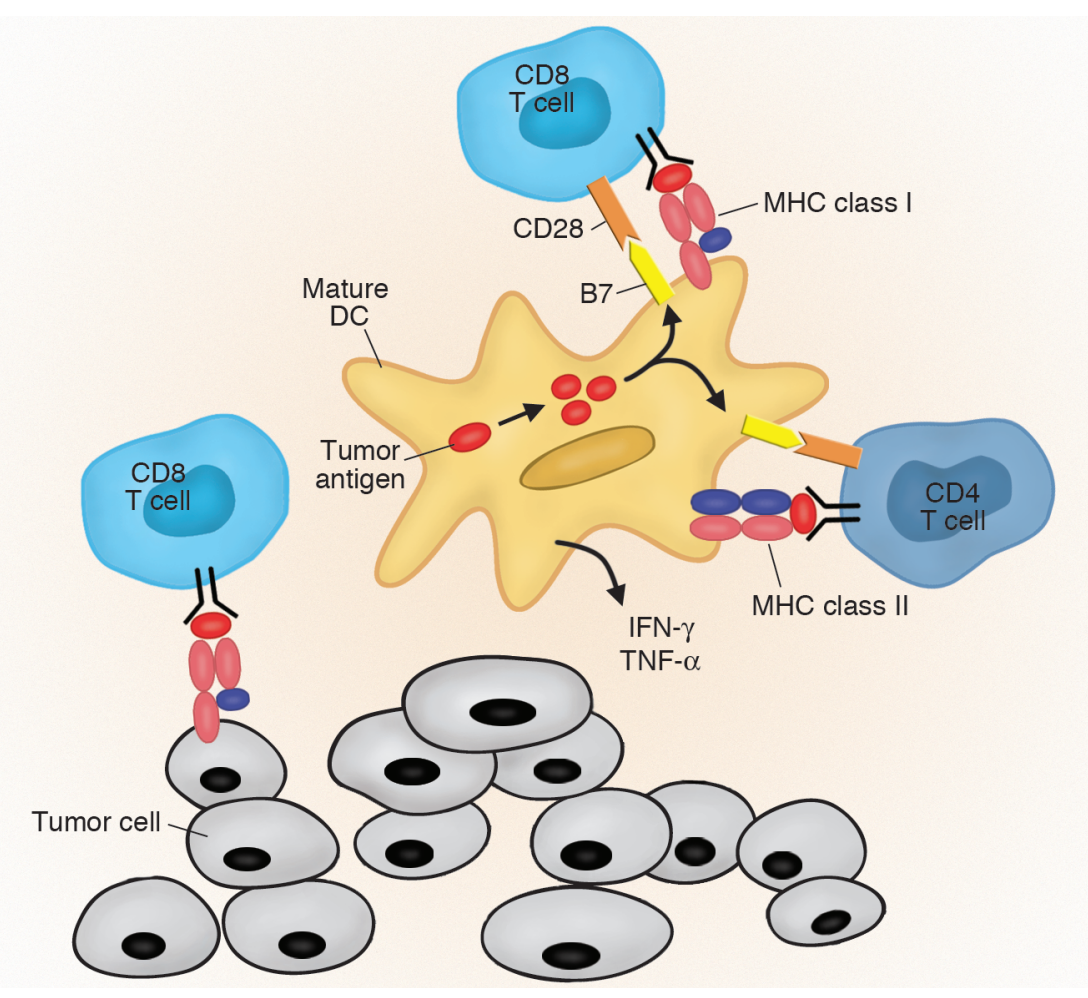


cancer vaccines may have clinical impact. Koutsky and colleagues have reported that immunization of young women with a vaccine targeting human papillomavirus (HPV) 16 protects not only against the development of HPV infection, but most likely against the development of cervical intraepithelial neoplasia (15). Furthermore, a recent randomized Phase III trial demonstrated that patients with melanoma whose disease had been completely resected and who bore specific HLA immune phenotypes could be immunized with a cell lysate-based vaccine, resulting in a significant survival benefit (16). The deliberate manipulation of local immune responses through the use of highly specific molecules and delivery vectors has been able to promote immunemediated regression of established and poorly immunogenic tumors in mice, as evidenced in the article by Furumoto and colleagues in this issue of the JCI (10). This represents an exciting new strategy for the treatment of human cancer and warrants renewed interest in clinical trials to determine the full potential for these approaches in cancer patients.

\section{Acknowledgments}

This work was supported by grants from the NIH (KO8CA79881) and the Doris Duke
Charitable Foundation (T98052) (to Howard L. Kaufman), and from the National Cancer Institute (K24CA85218) (to Mary L. Disis). The authors also thank Ken Flanagan for assistance with graphics.

Address correspondence to: Howard L. Kaufman, Department of Surgery, Columbia University Medical Center, 177 Fort Washington Avenue MHB-7SK, New York, New York 10032, USA. Phone: (212) 342-6042; Fax: (212) 342-0234; E-mail: hlk2003@columbia.edu.

1. Finn, O.J. 2003. Cancer vaccines: between the idea and the reality. Nat. Rev. Immunol. 3:630-641.

2. Marincola, F.M., Wang, E., Herlyn, M., Seliger, B., and Ferrone, S. 2003. Tumors as elusive targets of T-cell-based active immunotherapy. Trends Immunol. 24:335-342.

3. Tatsumi, T., et al. 2002. Disease-associated bias in T helper type 1 (Th1)/Th2 CD4(+) T cell responses against MAGE-6 in HLA-DRB10401(+) patients with renal cell carcinoma or melanoma. J. Exp. Med. 196:619-628.

4. Liyanage, U.K., et al. 2002. Prevalence of regulatory $\mathrm{T}$ cells is increased in peripheral blood and tumor microenvironment of patients with pancreas or breast adenocarcinoma. J. Immunol. 169:2756-2761.

5. Somasundaram, R., et al. 2002. Inhibition of cytolytic $\mathrm{T}$ lymphocyte proliferation by autologous CD4+/CD25+ regulatory $\mathrm{T}$ cells in a colorectal carcinoma patient is mediated by transforming growth factor-beta. Cancer Res. 62:5267-5272.

6. Ramirez-Montagut, T., Turk, M.J., Wolchok, J.D., Guevara-Patino, J.A., and Houghton, A. 2003. Immunity to melanoma: unraveling the relation of tumor immunity and autoimmunity. Oncogene
22:3180-3187.

7. Pardoll, D. 2003. Does the immune system see tumors as foreign or self? Annu. Rev. Immunol. 21:807-839.

8. Morse, M.A., Mosca, P., Clay, T.M., and Lyerly, H.K. 2002. Dendritic cell maturation in active immunotherapy strategies. Expert Opin. Biol. Ther. 2:35-43.

9. Steinman, R.M. 2003. The control of immunity and tolerance by dendritic cell. Pathol. Biol. (Paris) 51:59-60.

10. Furumoto, K., Soares, L., Engleman, E.G., and Merad, M. 2004. Induction of potent antitumor immunity by in situ targeting of intratumoral DCs. J. Clin. Invest. 113:774-783. doi:10.1172/JCI200419762.

11. Sallusto, F., and Lanzavecchia, A. 2000. Understanding dendritic cell and T-lymphocyte traffic through the analysis of chemokine receptor expression. Immunol. Rev. 177:134-140.

12. Moser, B., and Loetscher, P. 2001. Lymphocyte traffic control by chemokines. Nat. Immunol. 2:123-128.

13. Giovarelli, M., et al. 2000. Tumor rejection and immune memory elicited by locally released LEC chemokine are associated with an impressive recruitment of APCs, lymphocytes, and granulocytes. J. Immunol. 164:3200-3206.

14. van Deventer, H.W., et al. 2002. Transfection of macrophage inflammatory protein 1 alpha into B16F10 melanoma cells inhibits growth of pulmonary metastases but not subcutaneous tumors. J. Immunol. 169:1634-1639.

15. Koutsky, L.A., et al. 2002. A controlled trial of a human papillomavirus type 16 vaccine. N. Engl. J. Med. 347:1645-1651.

16. Sosman, J.A., et al. 2002. Adjuvant immunotherapy of resected, intermediate-thickness, node-negative melanoma with an allogeneic tumor vaccine: impact of HLA class I antigen expression on outcome. J. Clin. Oncol. 20:2067-2075. 